ORIGINAL ARTICLE

\title{
Randomised controlled trial of swaddling versus massage in the management of excessive crying in infants with cerebral injuries
}

\author{
S Ohgi, T Akiyama, K Arisawa, K Shigemori
}

Arch Dis Child 2004;89:212-216. doi: 10.1136/adc.2002.025064

See end of article for authors' affiliations

....................

Correspondence to: Dr S Ohgi, Departments of Preventive Medicine \& Health Promotion, Nagasaki University School of Medicine, 1-12-4 Sakamoto, Nagasaki 852-8523, Japan; ohgi@

net2.nagasaki-u.ac.jp

Accepted 5 July 2003
Background: Infants with neonatal cerebral insults are susceptible to excessive crying as a result of difficulties with self-regulation.

Aims: To compare the effectiveness of swaddling versus massage therapy in the management of excessive crying of infants with cerebral insults.

Methods: Randomised three-week parallel comparison of the efficacy of two intervention methods. Infants with symptoms of troublesome crying and their parents were randomly assigned to a swaddling intervention group $(n=13)$ or a massage intervention group $(n=12)$.

Results: The amount of total daily crying decreased significantly in the swaddling group, but did not decrease significantly in the massage group. Infant behavioural profiles and maternal anxiety levels improved significantly in the swaddling group post-intervention. Parents in the swaddling group were more satisfied with the effectiveness of the intervention in reducing crying than parents in the massage group.

Conclusion: Results indicate that swaddling may be more effective than massage intervention in reducing crying in infants with cerebral injuries.
1 fants with brain lesions are easily over-stimulated and have difficulty in dealing with environmental stimuli, exhibiting neurobehavioural disorganisation including poor self-regulation. Such behaviour causes a dilemma for parents, as infants may be over-stimulated by normal nurturing behaviour such as rocking, touching, and other forms of physical interaction. Excessive crying, such as persistent inconsolable crying, causes enormous parental stress, which in turn affects the development of the parentinfant relationship. ${ }^{1-3}$ The cause of troublesome crying is not known, but it is presumed to be an excessive neurophysiological response caused by central nervous system (CNS) irritation or dysfunction to external and internal stimulation.

Several intervention studies have been carried out on healthy newborns with infantile colic. ${ }^{4-14}$ Parental counselling is beneficial, and is considered important in the current management of troublesome crying; it also provides an opportunity to support the infant-parent relationship. ${ }^{4-7}$ Infant massage has been suggested for the treatment of colicky infants and may become more widely used. ${ }^{8}$ In addition, massage therapy has been reported to promote growth and development in premature infants ${ }^{9}{ }^{10}$ and to improve early mother-infant interaction. ${ }^{11}$ However, other studies have found that infant massage is not effective for the treatment of infantile colic, when measured by the outcome of reduction in hours of crying. ${ }^{12}{ }^{13}$

Some previous studies have reported that swaddling has a calming, sleep promoting effect on infants..$^{14-17}$ Also, several studies of nursing care for premature infants have suggested that when infants are swaddled they spend more time in quiet sleep, exhibit fewer motor responses to stimuli, show more effective self-regulatory ability, and cry less than when unswaddled. ${ }^{18} 19$ It has been hypothesised that swaddling may effectively reduce crying by protecting the infant from external and internal stress. ${ }^{60}$ However, no studies have yet been carried out to determine whether swaddling similarly reduces excessive crying in infants with brain insults.
The aim of this study was to investigate the effects of swaddling versus massage therapy in reducing excessive crying of infants with brain lesions.

\section{METHODS \\ Subjects}

This study was conducted at the Department of Rehabilitation, Nagasaki University Hospital. A total of 62 infants aged less than 3 months after birth with a diagnosed and/or suspected brain lesion were recruited between January 2000 and April 2002. Eligibility criteria were: (1) postnatal age less than 3 months; (2) parental perception that the crying of the infant was bothersome; (3) at least three hours per day of crying; (4) singleton birth; and (5) no presence of epilepsy, feeding disability, respiratory disease, and/or atopic disease. Cerebral injuries (periventricular leukomalacia (PVL), intraventricular haemorrhage (IVH), or hypoxic-ischaemic encephalopathy (HIE) were documented by imaging techniques (computed tomography, magnetic resonance imaging), and supported by electroencephalograms (EEGs) and neurological examinations performed by paediatric and rehabilitation clinicians.

Among those enrolled, 35 families (56.5\%) perceived that their infants' excessive crying, which was defined as episodes of unexplained, persistent, and inconsolable crying, bothered the caretakers for at least one week. Eight of these families were excluded from this study because five infants had medical complications (three with a feeding disability, two with epilepsy, two with respiratory disease), two infants were twins, and one family relocated. One family of the residual 27

Abbreviations: NBAS, Neonatal Behavioral Assessment Scale; STAI, State-Trait Anxiety Inventory; HIE, hypoxic-ischaemic encephalopathy; PVL, periventricular leukomalacia; IVH, intraventricular haemorrhage; $\mathrm{SE}$, standard error 
withdrew from the study (refused to participate). Therefore, 26 families were enrolled in this study. Of the 26 participating infants randomised to the intervention groups, one infant in the massage group was excluded, because he developed a respiratory infection, which necessitated hospitalisation.

\section{Procedure}

This trial consisted of a randomised three-week parallel comparison of the efficacy of two intervention methods in the management of troublesome crying. Before starting the intervention, the parents were given an informed consent form and a one-week diary for recording the crying, sleeping, and feeding patterns of the infant. After a week, the diary was collected and the neurobehavioural examination of the infant and the maternal anxiety level was assessed preintervention. Then, the families were randomly assigned to a massage or to a swaddling group. Randomisation was performed according to the method of minimisation. ${ }^{21}$ The families started a three-week intervention programme according to the randomisation. Outpatient visits were made once a week to track the progress of intervention. On the completion of the trial the diaries were collected and the infants' neurobehavioural profiles and maternal anxiety levels were re-evaluated (post-intervention). In addition, the parents were interviewed to obtain their evaluation of the effectiveness of the given intervention. All participating families gave informed consent. The assignment of subjects was unknown by the investigator performing the evaluation of the effectiveness of the given intervention.

\section{Swaddling and massage methods}

The infants were wrapped in the following way: (1) The infant was placed on the cover blanket, lying on his back, wearing an undergarment and a diaper. Both upper arms were positioned on the precordium (mid-line). (2) Starting from the left side of the blanket, the blanket was wrapped around the whole body, working towards the right. (3) The tip of the feet portion was folded and placed over the infant's abdomen, covering the legs. At this point, the infants' legs were spontaneously flexed and abducted (in our study, minimal hip restraint was provided). (4) Taking the right side of the blanket, it was again wrapped around the whole body two or three times. (5) The infant's head was covered with the hood. It was recommended that the swaddling be used at least three times a day for at least 30 minutes each time. While children were awake, mother-infant interaction combined with sensory stimulation such as audiovisual and vestibular stimulation was recommended. If sleeping, maintenance of sleep was recommended.

At the beginning of the massage intervention, a physical therapist taught mothers the massage technique and gave them a brochure with written illustrated instructions. Massage included gentle stroking of the skin over the different parts of the head, body, and limbs. An attempt was made to maintain eye-to-eye contact as well as touch between the infant and the masseuse during the procedure. Massage was recommended three times a day for at least 15 minutes each time.

\section{Outcome measures \\ Diary}

The diary was based on those used in previous studies. ${ }^{12}{ }^{22}$ For one week pre-intervention, and for the last one week of the three-week intervention period, parents recorded the crying time, sleeping time, and feeding time at 15 minute intervals in the lower row of the diary. Parents were asked to complete time bars representing periods of inconsolability, including crying and/or fussing. Also, it included the time of parental behaviour (such as cuddling, rocking, etc) to calm the infant's crying during the infant's crying/fussing bouts. Parents were recommended to complete at least three records per week. The amount of daily crying time was calculated in terms of average time per day during each one-week interval (pre-intervention and last one week of the three-week intervention). The given interventions were recorded by drawing a line in the upper row. A massage was recorded when performed continuously for more than 15 minutes. Swaddling was recorded when carried out for more than 30 minutes.

\section{Neurobehavioural examination}

Neurobehavioural examination was assessed with the Neonatal Behavioral Assessment Scale (NBAS). ${ }^{23}$ The NBAS was performed by the same blinded NBAS certified examiner (TA). The NBAS scores were reduced to seven clusters: (1) habituation, (2) orientation, (3) motor performance, (4) range of state, (5) state regulation, and (6) autonomic stability.

\section{Maternal anxiety}

Maternal anxiety was measured using the State Anxiety Scale (Form X-1) of the Spielberger's State-Trait Anxiety Inventory (STAI), which has been commonly used to assess the anxiety of parents during paediatric procedures. ${ }^{24}$ This scale measures a transitory emotional response to a stressful situation and consists of 20 phrases with responses on a four point Likert scale.

\section{Parental evaluation}

Parental evaluation included two forms of evaluation of the perceived effects of the given intervention: whether it had an effect on reducing crying and whether it made the infant easier to handle. The effect of the intervention was evaluated using three categories: (1) not effective, (2) effective to some extent, or (3) effective.

\section{Statistical analysis}

Parents' and infants' characteristics were compared using the Mann-Whitney $U$ test and Student's $t$ test for continuous data and Fisher's exact test or $\chi^{2}$ test for discrete data. The paired $t$ test was used to analyse the difference between preand post-intervention in the daily amount of total crying, the cluster scores of the NBAS, and STAI score. The mean differences (post- minus pre-intervention) were calculated and compared between the two groups using Student's $t$ test. Results of the parental evaluation were analysed using Fisher's exact test; p values less than 0.05 (two-tailed tests) were regarded as statistically significant. All statistical analysis was performed using SPSS for Windows (version $10.7 \mathrm{~J})$ software.

\section{RESULTS}

\section{Background comparison}

Table 1 presents the characteristics of the study infants and those of their mothers. No differences between the groups were observed in infant and maternal factors. The diagnoses of those infants in the swaddling group were: four full term infants with hypoxic-ischaemic encephalopathy (HIE), six premature infants with periventricular leukomalacia (PVL), one premature infant with intraventricular haemorrhage (IVH), and two others (developmental delay). In the massage group, infants were diagnosed as follows: four with HIE, six with PVL, and two others. There was thus no difference in the distribution of diagnosis between the two groups. With regard to social factors, there were no single-parent families and no fathers who were out of work in either group. All mothers stayed at home to care for their children full-time during the study period. 


\begin{tabular}{|c|c|c|c|}
\hline & Swaddling & Massage & $\mathrm{p}$ value \\
\hline No. of subjects & 13 & 12 & \\
\hline Gender (M/F) & $10 / 3$ & $9 / 3$ & $1.00 \S$ \\
\hline Birth weight $(g)^{*}$ & $1580(1166-280)$ & $1379(765-150)$ & $0.31 \ddagger$ \\
\hline Low birth weight infants (yes) & & 6 & $0.70 \S$ \\
\hline Gestational age at birth (weeks) $\dagger$ & $33.4(5.3)$ & $34.3(5.6)$ & 0.819 \\
\hline Age on entry into trial after birth (weeks)† & $11.6(5.1)$ & $11.3(5.6)$ & $0.89{ }^{\circ}$ \\
\hline \multicolumn{4}{|l|}{ Apgar score } \\
\hline $1 \mathrm{~min} t$ & $3.6(2.0)$ & $4.3(1.9)$ & 0.68 \\
\hline $5 \mathrm{~min} \dagger$ & $5.5(2.2)$ & $5.9(1.8)$ & $0.96^{\circ}$ \\
\hline \multicolumn{4}{|l|}{ Diagnosis } \\
\hline HIE/PVL/IVH/other & $4 / 6 / 1 / 2$ & $4 / 5 / 1 / 2$ & $1.00 * *$ \\
\hline \multicolumn{4}{|l|}{ Feeding type before intervention } \\
\hline Mostly breast fed/mostly formula fed & $1 / 12$ & $2 / 10$ & $0.59 \S$ \\
\hline Maternal age $\dagger$ & $30.8(3.9)$ & $31.2(3.5)$ & $0.82^{\circ}$ \\
\hline Single mother (yes) & 0 & 0 & \\
\hline Siblings (yes) & 3 & 4 & $0.67 \S$ \\
\hline Maternal education (under high school) & 0 & 0 & \\
\hline \multicolumn{4}{|c|}{$\begin{array}{l}\text { HIE, hypoxic ischaemic encephalopathy; PVL, periventricular leukomalacia; IVH, intraventricular haemorrhag } \\
{ }^{*} \text { Median (range). } \\
\text { †Average (SD). } \\
\text { †Mann-Whitney U test. } \\
\text { SFisher's exact test. } \\
\text { - }\end{array}$} \\
\hline
\end{tabular}

\section{Diary}

Parental diary records were made a mean of 3.2 (standard error (SE) 0.2) times in the swaddling and 3.4 (SE 0.2) times in the massage group pre-intervention. Also, they were made a mean of 3.3 (SE 0.2) times in both groups during the last week of the intervention. There were no subjects with incomplete records.

\section{Amount of total daily crying}

Table 2 shows comparisons between the two groups of the amount of total daily crying. The mean amount of total daily crying pre-intervention was 10.7 (SE 0.7) hours/day in the massage group infants and 10.8 (SE 0.7) hours/day in the swaddling group infants. At the post-intervention stage, the mean amount of total daily crying was 10.2 (SE 0.7) hours/day in the massage group infants and 7.7 (SE 0.6) hours/day in the swaddling group infants. The amount of total daily crying decreased significantly in the swaddling group, but not in the massage group infants, by the postintervention stage. The mean difference of the total amount of crying was 0.5 (SE 0.8) hours/day in the massage group and 3.0 (SE 0.6) hours/day in the swaddling group.

\section{Interventions}

Massage was performed a mean of 3.3 (SE 0.2) times a day and the swaddling was used 3.4 (SE 0.2) times a day during the three-week intervention. The mean duration of the

Table 2 The amount of total daily crying pre- and postintervention

\begin{tabular}{lll}
\hline & Swaddling & Massage \\
\hline Pre-intervention & $10.8(0.7)$ & $10.7(0.7)$ \\
Post-intervention & $7.7(0.6)^{*}$ & $10.2(0.7)$ \\
Mean difference & $-3.1(0.5) \dagger$ & $-0.5(0.8)$ \\
\hline
\end{tabular}

Results expressed as mean (SE).

*Significantly different between pre- and post-intervention. The amount of total daily crying decreased significantly in the swaddling group $(p<0.01)$, but not in the massage group.

†Significantly different between the two groups. The mean difference of the amount of total daily crying was significantly greater in the swaddling group than in the massage group $(p=0.01)$. intervention was 0.8 (SE 0.1 ) hours/day in the massage group and 4.2 (SE 0.3) hours/day in the swaddling group.

\section{The NBAS cluster scores}

Table 3 shows the NBAS behavioural cluster scores for both groups, pre- and post-intervention. Post-intervention orientation, state range, and state regulation cluster scores were significantly improved in the swaddling group, but not in the massage group.

\section{The STAI score}

Table 4 shows the STAI score for both groups, pre- and postintervention. The STAI score improved significantly in the swaddling group, but not in the massage group.

\section{Parental evaluation}

Table 5 shows the results of the parental evaluation of the effect of intervention. Parents in the swaddling group were more satisfied with the effects of the intervention than parents in the massage group. Eight $(61.5 \%)$ of the parents in the swaddling group compared with only two (16.7\%) of the parents in the massage group reported that crying decreased as a result of the intervention. Also, in terms of parental evaluation of whether the intervention made the infant easier to handle (reciprocal effect), parents in the swaddling group gave more positive responses than parents in the massage group. Seven $(53.8 \%)$ of the parents in the swaddling group and only one $(8.3 \%)$ of the parents in the massage group reported that handling of their infant became easier.

\section{DISCUSSION}

Although a number of intervention studies on normal, mature infants with colicky crying have been conducted, there is a dearth of research on the management of excessive crying with cerebral insults. The rationale of our study was that swaddling may be more effective in protecting infants with cerebral injuries from external and internal stimuli, thus reducing crying in these infants. The purpose of this study was to test this hypothesis by comparing two intervention methods, massage and swaddling, over a period of three weeks. In the swaddling group, the total amount of postintervention crying significantly decreased by $28 \%$ compared with the level of pre-intervention crying. This decrease in 
Table 3 NBAS cluster scores pre- and post-intervention

\begin{tabular}{|c|c|c|c|c|c|c|}
\hline & \multicolumn{3}{|l|}{ Swaddling } & \multicolumn{3}{|l|}{ Massage } \\
\hline & Pre-intervention & Post-intervention & Mean difference & Pre-intervention & Post-intervention & Mean difference \\
\hline Habituation & $6.7(0.2)$ & $7.0(0.2)$ & $0.3(0.2)$ & $6.9(0.3)$ & $6.9(0.2)$ & $0.1(0.2)$ \\
\hline Orientation & $4.0(0.3)$ & $4.9(0.4)^{*}$ & $0.9(0.3) \dagger$ & $4.0(0.4)$ & $4.2(0.4)$ & $0.2(0.3)$ \\
\hline Motor & $3.5(0.2)$ & $4.0(0.3)$ & $0.5(0.3)$ & $3.4(0.3)$ & $3.5(0.3)$ & $0.1(0.2)$ \\
\hline State range & $3.5(0.2)$ & $3.9(0.2)^{*}$ & $0.4(0.2) \dagger$ & $3.4(0.2)$ & $3.4(0.3)$ & $0.0(0.2)$ \\
\hline State regulation & $3.5(0.2)$ & $4.0(0.3)^{*}$ & $0.5(0.2) \dagger$ & $3.4(0.2)$ & $3.6(0.2)$ & $0.2(0.2)$ \\
\hline Autonomic stability & $6.3(0.2)$ & $6.6(0.2)$ & $0.3(0.3)$ & $6.4(0.3)$ & $6.5(0.2)$ & $0.1(0.3)$ \\
\hline
\end{tabular}

Results expressed as mean (SE).

*Significantly different between pre- and post-intervention. Orientation $(p=0.01)$, state range $(p=0.02)$, and state regulation ( $p=0.01)$ cluster scores recovered significantly in the swaddling group.

†Significantly different between the two groups. The mean difference of the orientation $(p=0.05)$, state range $(p=0.02)$, and state regulation ( $p=0.03)$ cluster scores were significantly higher in the swaddling group than in the massage group.

crying was considered substantial, even in comparison to infants who received massage intervention. These results indicate that swaddling may suppress crying by protecting infants from external and internal stimulation. The decrease of crying in swaddling group infants would reflect the effect of the intervention in itself rather than the natural course of infant crying, because there was no significant difference in maturational age between the two groups, and crying time did not decrease in infants of the massage group. However, as there are very few systematic studies of time related change in crying in infants with cerebral lesions, we need longer follow up studies.

The results given by the NBAS also showed that infants who received the swaddling intervention improved their abilities to: (1) maintain an alert state and to focus on attention-orientation tasks; (2) regulate behavioural states; and (3) cope with stress. These results suggest that swaddling may improve the neurobehavioural organisation in infants with brain lesions. These findings are supported by the literature on swaddling for premature infants. ${ }^{18} 19$ In addition, results of the maternal STAI showed that the maternal anxiety perception in the swaddling group was improved over the three-week intervention period. Also, in terms of the parental evaluation, the rates of reduction in crying and of improvement in ease of handling were higher in the swaddling group. Reduction of crying by swaddling will probably help to alleviate parental anxiety and stress. In essence, short term changes in an infant's behaviour and in parental mental health, in response to swaddling, can serve to initiate a positive cycle of interaction between parents and infants that may have a long term impact on the child development outcome.

In considering the effects of massage therapy, no clear benefit in the massage group was observed in this study. Massage is commonly used as a method of consoling, and has even been recommended for treatment of colicky infants. However, Huhtala et al found that massage as an intervention for colicky infants does not modify the natural course of early infant crying and colic. ${ }^{12}$ In this study, we obtained similar

Table 4 STAl scores pre- and post-intervention

\begin{tabular}{lll}
\hline & Swaddling & Massage \\
\hline Pre-intervention & $44.3(2.3)$ & $44.2(2.4)$ \\
Post-intervention & $39.2(2.0)^{*}$ & $44.8(2.2)$ \\
Mean difference & $-5.1(1.9)^{\dagger}$ & $0.7(2.1)$ \\
\hline
\end{tabular}

Results expressed as mean (SE).

*Significantly different between pre- and post-intervention. The STAI score improved significantly in the swaddling group $(p=0.01)$.

†Significantly different between the two groups. The mean difference of the STAI score was significantly greater in the swaddling group than in the massage group $(p=0.04)$. results on infants with cerebral insults. In our study the mean duration of massage was shorter than a swaddling intervention. It is conceivable that massage performed for such a short period of time may not be effective. In order to promote sensory-motor development in infants with brain lesions, sensory stimulation to an appropriate degree, must be reinforced. In terms of future directions for research, it may be rewarding to evaluate the efficacy of combining swaddling and sensory-motor interventions.

In our study, the average amount of total crying per day in infants with cerebral insults who cried excessively, was approximately 11 hours. Brazelton studied 80 healthy newborns and found that crying gradually increased to a peak daily median of 2.75 hours at 6 weeks of age. ${ }^{25}$ The amount of total crying of the target infants in our study was much greater than that reported by Brazelton. One possibility could account for the fact that the time of parental behaviour to calm the infant's crying during the infant's crying/fussing bouts was included in the crying time. However, infants with brain lesions are easily over-stimulated, and are susceptible to a rapid state change to crying. Also, as these infants have difficulties with self-regulation and inconsolable crying, parents need to intervene more frequently to calm their babies. We believe the amounts of crying that parents reported in this study approximate the true number of hours that infants cried. In addition, we used the "15 minute rule" for the diary recordings, which may affect the sensitivity of the measures of amount of crying reported. Previous study of parental diaries has suggested that the "5 minute rule" may provide a more sensitive estimate of the accuracy of diaries. ${ }^{26}$ Furthermore, future study should include an objective analysis using 24-hour audiotaped recording.

In conclusion, swaddling appears to be more effective than massage in reducing excessive crying in infants with cerebral insults. Swaddling is associated with an improved neurobehavioural organisation and reduced maternal anxiety, which may ultimately promote parent-infant interaction. The effectiveness of swaddling in reducing excessive crying

Table 5 Parental evaluation of the effect of intervention

\begin{tabular}{llll}
\hline & Swaddling & Massage & p value* $^{*}$ \\
\hline Crying decrease & & & \\
$\quad$ Not effective & $1(7.7)$ & $6(50.0)$ & \\
$\quad$ Effective to some extent & $4(30.8)$ & $4(33.3)$ & \\
$\quad$ Effective & $8(61.5)$ & $2(16.7)$ & 0.03 \\
Easier to handling & & & \\
$\quad$ Not effective & $2(15.4)$ & $6(50.0)$ & \\
$\quad$ Effective to some extent & $4(30.8)$ & $5(41.7)$ & \\
$\quad$ Effective & $7(53.8)$ & $1(8.3)$ & 0.04 \\
\hline
\end{tabular}

Results expressed as no. (\%).

${ }^{*} \chi$ test. 
may be related to the protection of the infant from external and internal stimulation.

\section{Authors' affiliations}

S Ohgi, K Arisawa, Department of Preventive Medicine \& Health Promotion, Nagasaki University School of Medicine, Nagasaki, Japan T Akiyama, K Shigemori, Department of Rehabilitation, Nagasaki University Hospital, Nagasaki, Japan

\section{REFERENCES}

1 Beebe SA, Casey R, Pinto-Martin J. Association of reported infant crying and maternal parenting stress. Clin Pediatr (Phila) 1993;32:15-19.

2 St James-Roberts I, Conroy S, Wilsher K. Links between maternal care and persistent infant crying in the early months. Child Care Health Dev 1998;24:353-76.

3 Miller AR, Barr RG, Eaton WO. Crying and motor behavior of six-week-old infants and postpartum maternal mood. Pediatrics 1993:92:551-8.

4 Wolke D, Gray P, Meyer R. Excessive infant crying: a controlled study of mothers helping mothers. Pediatrics 1994;94:322-32.

5 Lucassen PLB, Assendelff WJJ, Gubbels JW, et al. Effectiveness of treatments or infantile colic: systematic review. BMJ 1998:316:1563-9.

6 Garrison MM, Christakis DA. A systematic review of treatments for infant colic. Pediatrics 2000;106:184-90.

7 Raiha H, Lehtonen L, Huhtala V, et al. Excessively crying infant in the family: mother-infant, father-infant and mother-father interaction. Child Care Health Dev 2002;28:419-29.

8 Larsen J-H. Infants' colic and belly massage. Practitioner 1990;234:396-7.

9 Rice RD. Neurophysiological development in premature infants following stimulation. Dev Psychol 1977;13:69-76.

10 Field TM, Schanberg SM, Scafidi F. Tactile/kinesthetic stimulation effects on preterm neonates. Pediatrics 1986;77:654-8.
11 White-Traut RC, Nelson MN. Maternally administered tactile, auditory, visual, and vestibular stimulation: relationship to later interactions between mothers and premature infants. Res Nurs Health 1988;11:31-9.

12 Huhtala V, Lehtonen L, Heinonen R, et al. Infant massage compared with crib vibrator in the treatment of colicky infants. Pediatrics 2000;105:6-12.

13 Olafsdottira E, Forsheib S, Flugea G, et al. Randomised controlled trial of infantile colic treated with chiropractic spinal manipulation. Arch Dis Child 2001;84:138-41.

14 Lipton EL, Steinschneider A, Richmond JB. Swaddling, a child care practice: historical, cultural, and experimental observations. Pediatrics 1965;35:521-67.

15 Brackbill Y. Continuous stimulation reduces arousal level: stability of effect over time. Child Dev 1973:44:43-6.

16 Caglayan S, Yaprak I, Sackin E, et al. A different approach to sleep problems of infancy: swaddling above the waist. Turk J Pediatr 1991;33:1 17-20.

17 Giacoman SL. Hunger and motor restraint on arousal and visual attention in the infant. Child Dev 1971:42:605-14.

18 Short MA, Brooks-Brunn JA, Reeves DS, et al. The effects of swaddling versus standard positioning on neuromuscular development in very low birth weight infants. Neonatal Network 1996;15:25-31.

19 Neu M, Browne JV. Infant physiologic and behavioral organization during swaddled versus unswaddled weighing. Perinatology 1997;17:193-8.

20 McKenzie S. Troublesome crying in infants: effect of advice to reduce stimulation. Arch Dis Child 1991;66:1416-20.

21 Traves DR. Minimization: a new method of assigning patients to treatment and control groups. Clin Pharmacol Ther 1974;15:443-53.

22 Lehtonen L, Korvenranta H. Infantile colic. Seasonal incidence and crying profiles. Arch Pediatr Adolesc Med 1995; 149:533-6.

23 Brazelton TB, Nugent JK. Neonatal behavioral assessment scale, 3rd edn. CDM No. 137. Cambridge, MA: Cambridge University Press, 1995.

24 Spielberg CD, Gorsuch RL, Lushene RE. STAl manual. Palo Alto, CA: Consulting Psychologist Press, 1970:23-49.

25 Brazelton TB. Crying in infancy. Pediatrics 1962;29:579-88.

26 Barr RG, Kramer MS, Boisjoly C, et al. Parental diary of infant cry and fuss behaviour. Arch Dis Child 1988;63:380-7. 